\title{
ANGLE GEOMETRY IN THE UNIVERSAL TEICHMÜLLER SPACE
}

\author{
JINHUA FAN AND YUNPING JIANG
}

(Communicated by Yingfei Yi)

\begin{abstract}
In this paper we investigate angle geometry in the universal Teichmüller space. We construct three examples of triangles bounded by geodesic segments such that the first example has the sum of the three inner angles less than $\pi$, the second example has the sum of the three angles equal to $\pi$, and the third example has the sum of the three angles greater than $\pi$. Our result gives a negative answer to a problem raised by Zhong Li and Yi Qi. Moreover, our results indicate that the universal Teichmüller space presents all hyperbolic, Euclidean, and spherical phenomena in angle geometry.
\end{abstract}

\section{INTRODUCTION}

First let us recall the definition of the universal Teichmüller space. Instead of using the open unit disk $\mathbb{D}=\{z \in \mathbb{C}|| z \mid<1\}$ in the complex plane in the definition, we use the strip

$$
S=\{z=x+i y \in \mathbb{C} \mid-\infty<x<+\infty, 0<y<1\} .
$$

Suppose $\mathcal{M}(S)$ is the open unit ball of $L^{\infty}(S)$. For every $\mu \in \mathcal{M}(S)$, we consider the Beltrami equation

$$
f_{\bar{z}}(z)=\mu(z) f_{z}(z)
$$

We use $f^{\mu}$ to denote the solution of the Beltrami equation which is a quasiconformal self-mapping of $S$ and normalized by $0, i$ and $\infty$ fixed. Here $\mu \in \mathcal{M}(S)$ is called a Beltrami coefficient. Two Beltrami coefficients $\mu, \nu \in M(S)$ are said to be equivalent, denoted by $\mu \sim \nu$, if $\left.f^{\mu}\right|_{\partial S}=\left.f^{\nu}\right|_{\partial S}$. We use $[\mu]$ or $\left[f^{\mu}\right]$ to denote the equivalence class of $\mu$. Then the universal Teichmüller space

$$
T(S)=\{[\mu] \mid \mu \in \mathcal{M}(S)\}
$$

Received by the editors February 21, 2013 and, in revised form, August 30, 2013.

2010 Mathematics Subject Classification. Primary 30C75, 30F60.

Key words and phrases. Teichmüller distance, geodesic segment, inner angle.

The first author was partially supported by a grant from the NSF of China (No. 11201228 and No. 11371194).

The second author was partially supported by the Collaboration Grant from the Simons Foundation (No. 199837), the CUNY Collaborative Incentive Research Grant (No. 1861), awards from PSC-CUNY, a grant from the NSF of China (No. 11171121), and a collaboration grant from Academy of Mathematics and Systems Science and the Morningside Center of Mathematics at the Chinese Academy of Sciences. 
is the space of all equivalence classes. We know that $T(S)$ is an infinite dimensional complex Banach manifold with Teichmüller distance

$$
d_{T}([\mu],[\nu])=\frac{1}{2} \inf _{\eta \in\left[f^{\nu} \circ\left(f^{\mu}\right)^{-1}\right]} \log \frac{1+\|\eta\|_{\infty}}{1-\|\eta\|_{\infty}}, \quad \forall[\mu],[\nu] \in T(S) .
$$

Definition 1. A Beltrami coefficient $\mu \in \mathcal{M}(S)$ is extremal if

$$
\|\nu\|_{\infty} \geq\|\mu\|_{\infty}, \quad \forall \nu \in[\mu] .
$$

We say that $\mu$ is uniquely extremal if

$$
\|\nu\|_{\infty}>\|\mu\|_{\infty}, \quad \forall \nu \neq \mu \in[\mu] .
$$

The corresponding map $f^{\mu}$ is called an extremal quasiconformal mapping if $\mu$ is extremal.

The reader can refer to [4,5,8, for further properties of the universal Teichmüller space, and to [1,6, 15, 17] for theories of extremal quasiconformal mappings.

Suppose $A=[\mu]$ and $B=[\nu]$ are two points in $T(S)$. Let $\eta \in\left[f^{\nu} \circ\left(f^{\mu}\right)^{-1}\right]$ be an extremal Beltrami coefficient. Then

$$
\left\{\left[f^{t \eta} \circ f^{\mu}\right] \mid 0 \leq t \leq 1\right\}
$$

is a geodesic connecting $A$ and $B$. For any two points $A=[\mu]$ and $B=[\nu]$ in $T(S)$, we use $\gamma_{A B}$ to denote a geodesic connecting $A$ and $B$. For a given geodesic $\gamma_{A B}$, we use $\gamma_{B A}$ to denote the geodesic segment which is same as $\gamma_{A B}$ but with the starting point $B$ and the endpoint $A$. Since there may be many geodesic segments between $A$ and $B$ in $T(S)$, it is worth noting that $\gamma_{A B}$ and $\gamma_{B A}$ may be not of the form (2) (refer to [3,9]). Furthermore, that $\gamma_{A B}$ is of the form (2) does not imply that $\gamma_{B A}$ is of the form (2), and vise versa. However, in this paper, when we talk about a geodesic between $A=[\mu]$ and $B=[\nu]$, we will mean $\gamma_{A B}$ or $\gamma_{B A}$ in the form of (2).

For a given geodesic segment $\gamma_{A B}$, let $\gamma_{A B}(r)$ be the point in $\gamma_{A B}$ such that the Teichmüller distance between $A$ and $\gamma_{A B}(r)$ is

$$
d_{T}\left(\gamma_{A B}(r), A\right)=\frac{1}{2} \log \frac{1+r}{1-r} .
$$

For more properties of the geodesic geometry in the universal Teichmüller space, the reader can refer to $3,9,13$.

Suppose $A=[\mu], B=[\nu]$, and $C=[\eta]$ are three points in $T(S)$. Let $\gamma_{A B}, \gamma_{A C}$, and $\gamma_{B C}$ be the geodesic segments connecting $A$ and $B, A$ and $C$, and $B$ and $C$, respectively. In this paper, we say $\gamma_{A B}, \gamma_{A C}$ and $\gamma_{B C}$ form a triangle in $T(S)$ which we denote as

$$
\triangle A B C,
$$

if $\gamma_{A B}$ or $\gamma_{B A}, \gamma_{A C}$ or $\gamma_{C A}$, and $\gamma_{B C}$ or $\gamma_{C B}$ are in the form of (2).

Definition 2. We are given a triangle in the form of (3). The angle at $A$, denoted as $\angle A$, is defined as

$$
\begin{aligned}
\angle A & =2 \arcsin \left\{\begin{array}{l}
\frac{1}{2} \lim _{r \rightarrow 0} \frac{d_{T}\left(\gamma_{A B}(r), \gamma_{A C}(r)\right)}{d_{T}\left(\gamma_{A B}(r), A\right)} \\
=2 \arcsin \left\{\frac{1}{2} \lim _{r \rightarrow 0} \frac{d_{T}\left(\gamma_{A B}(r), \gamma_{A C}(r)\right)}{r}\right.
\end{array}\right\}
\end{aligned}
$$

if the limit exists. Similarly, we can define $\angle B$ and $\angle C$. 
The definition of an angle was given in [14] when $A=[0]$. The above definition is more general. In the definition, the existence of the limit for a general triangle is still a problem. For a finite dimensional Teichmüller space, Yao has claimed that the limit always exists; see [19. For a general Teichmüller space, Li and Qi in [14] used to give a sufficient condition for the existence of the limit. The following problem is raised from the paper [14].

Problem 1. Suppose the three inner angles $\angle A, \angle B$, and $\angle C$ of a triangle $\triangle A B C$ in the form of (3) exist. Is the sum $\angle A+\angle B+\angle C$ of the three inner angles always less than $\pi$ ?

In this paper, we give a negative answer to this problem when the Teichmüller space is the universal Teichmüller space. We do not only give a negative answer to the problem but we also prove that all cases can happen in the universal Teichmüller space $T(S)$. More precisely, We prove the following three theorems.

Theorem 3. There exists a triangle $\triangle A B C$ in the form of (3) in $T(S)$ such that

$$
\angle A+\angle B+\angle C<\pi \text {. }
$$

Theorem 4. There exists a triangle $\triangle A B C$ in the form of (3) in $T(S)$ such that

$$
\angle A+\angle B+\angle C=\pi .
$$

Theorem 5. There exists a triangle $\triangle A B C$ in the form of (3) in $T(S)$ such that

$$
\angle A+\angle B+\angle>\pi \text {. }
$$

We will give detailed proofs in the next three sections. We will keep the notation $z=x+i y$.

\section{Proof of Theorem 3}

Suppose $\varphi(z)$ is a Strebel differential, which means that $\left[t \frac{\bar{\varphi}}{|\varphi|}\right]$ is a Strebel point for any $t \in \mathbb{D}$. For the definition and properties of a Strebel point, we refer to [2] and [7]. Consider the Beltrami coefficient

$$
\mu_{t}=t \frac{\bar{\varphi}}{|\varphi|}
$$

Then $D_{\varphi}=\left\{\left[\mu_{t}\right] \mid t \in \mathbb{D}\right\}$ is a Teichmüller disk in $T(S)$. Consider the hyperbolic disk $\mathbb{D}$ with the hyperbolic metric

$$
d \rho=\frac{|d z|}{1-|z|^{2}} .
$$

Then the map $\alpha(t)=\left[\mu_{t}\right]: \mathbb{D} \rightarrow D_{\varphi}$ is an isometry.

Let $A=[0] ; B=\left[\mu_{1 / 2}\right]$ and $C=\left[\mu_{i / 2}\right]$. Also let $\triangle A B C$ be the geodesic triangle in the Teichmüller disk $D_{\varphi}$. We claim that $\triangle A B C$ is in the form of (3).

Since $\mu_{1 / 2}$ is uniquely extremal with constant modular $\left|\mu_{1 / 2}\right|=1 / 2$, by the result in [3], the geodesic segment $\gamma_{A B}$ connecting $A$ and $B$ in $T(S)$ is unique. For the same reason, the geodesic segment $\gamma_{A C}$ connecting $A$ and $C$ in $T(S)$ is unique too. The Beltrami coefficient for $\left(f^{\mu_{1 / 2}}\right)^{-1}$ can be calculated as

$$
\mu_{\left(f^{\mu_{1 / 2}}\right)^{-1}}=-\frac{1}{2} \frac{\bar{\varphi}}{|\varphi|} \frac{f_{z}^{\mu_{1 / 2}}}{\overline{f_{z}^{\mu_{1 / 2}}}} \circ\left(f^{\mu_{1 / 2}}\right)^{-1} .
$$


Similarly, the Beltrami coefficient for $f^{\mu_{i / 2}} \circ\left(f^{\mu_{1 / 2}}\right)^{-1}$ can be calculated as

$$
\mu_{f^{\mu_{i / 2}} \circ\left(f^{\mu_{1 / 2}}\right)^{-1}}=\frac{\mu_{i / 2}-\mu_{1 / 2}}{1-\mu_{i / 2} \overline{\mu_{1 / 2}}} \frac{f_{z}^{\mu_{1 / 2}}}{\overline{f_{z}^{\mu_{1 / 2}}}} \circ\left(f^{\mu_{1 / 2}}\right)^{-1}=\frac{2(i-1)}{4-i} \frac{\bar{\varphi}}{|\varphi|} \frac{f_{z}^{\mu_{1 / 2}}}{\overline{f_{z}^{\mu_{1 / 2}}}} \circ\left(f^{\mu_{1 / 2}}\right)^{-1} .
$$

Since $\left[f^{\mu_{1 / 2}}\right]$ is a Strebel point, its inverse $\left(f^{\mu_{1 / 2}}\right)^{-1}$ is also a Strebel point. This implies that there is another holomorphic function $\varphi^{*}$ on $S=f^{\mu_{1 / 2}}(S)$ with $\left\|\varphi^{*}\right\|=$ 1 such that

$$
\mu_{\left(f^{\left.\mu_{1 / 2}\right)^{-1}}\right.}=\frac{1}{2} \frac{\overline{\varphi^{*}}}{\left|\varphi^{*}\right|}
$$

This implies that

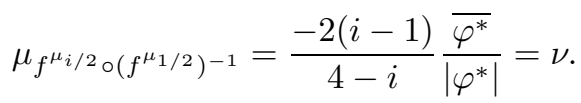

Since $\left\|\varphi^{*}\right\|=1, \nu$ is uniquely extremal with constant modular. So the geodesic segment $\gamma_{B C}$ connecting $B$ and $C$ is unique. We concluded that the geodesic triangle $\triangle A B C$ in the Teichmüller disk $D_{\varphi}$ is in the form of (3).

Remember that $\alpha(t)$ is an isometry between the hyperbolic disk $\mathbb{D}$ and the Teichmüller disk $D_{\varphi}$. Consider the hyperbolic triangle $\triangle a b c$ in $\mathbb{D}$ for $a=0, b=1 / 2$ and $c=i / 2$. Then $\alpha(\triangle a b c)=\triangle A B C$. Thus $\angle A, \angle B$ and $\angle C$ in $\triangle A B C$ in $T(S)$ is equal to $\angle a, \angle b$ and $\angle c$ in $\triangle a b c$ in $\mathbb{D}$ respectively. Since the angle sum of any triangle in the hyperbolic plane $\mathbb{D}$ is less than $\pi$. So

$$
\angle a+\angle b+\angle c<\pi
$$

in the triangle $\triangle a b c$. This implies that

$$
\angle A+\angle B+\angle C<\pi
$$

in the triangle $\triangle A B C$. This completes the proof.

Remark 6 . From the proof of this theorem, we actually proved that Theorem 3 holds for any Teichmüller space.

\section{Proof of Theorem 4}

Let us recall a lemma from 18 .

Lemma 7. Suppose $E$ is a subset in $\partial S$. Let $F_{K}(x, y)=K x+i y$, where $K$ is a positive constant. Consider

$Q\left(F_{K}, E\right)=\left\{f \mid f\right.$ is a quasiconformal mapping of $S$ onto $\left.F_{K}(S),\left.f\right|_{E}=\left.F_{K}\right|_{E}\right\}$.

Then $F_{K}$ is extremal in $Q\left(F_{K}, E\right)$, that is,

$$
\left\|\mu_{F_{K}}\right\|_{\infty} \leq\left\|\mu_{f}\right\|_{\infty}, \quad \forall f \in Q\left(F_{K}, E\right),
$$

if at least one of the points $\pm \infty$ is an accumulation point of $E$.

From Lemma 7, we have the following lemma.

Lemma 8. Define

$$
f(x, y)= \begin{cases}K_{1} x+i y, & (x, y) \in S, x \geq 0 \\ K_{2} x+i y, & (x, y) \in S, x<0\end{cases}
$$

where $K_{1}$ and $K_{2}$ are two positive constants. Then $\mu_{f}$ is extremal in $[f]$. 
Proof. Let $k_{1}=\frac{K_{1}-1}{K_{1}+1}$ and $k_{2}=\frac{K_{2}-1}{K_{2}+1}$; without loss of generality, we assume that $\left|k_{2}\right| \leq\left|k_{1}\right|$. Let $E=\{(x, y) \in \partial S \mid x \geq 0\}$ and let $F_{K_{1}}(x, y)=K_{1} x+i y$. Then $Q\left(F_{K_{1}}, E\right)=\left\{g \mid g\right.$ is a quasiconformal mapping from $S$ onto $\left.F_{K_{1}}(S),\left.g\right|_{E}=\left.F_{K_{1}}\right|_{E}\right\}$. By Lemma 7. $F_{K_{1}}$ is extremal in $Q\left(F_{K_{1}}, E\right)$. Since $f \in Q\left(F_{K_{1}}, E\right)$ and since $\left\|\mu_{f}\right\|_{\infty}=\left\|\mu_{F_{K_{1}}}\right\|_{\infty}=k_{1}, f$ is extremal in $Q\left(F_{K_{1}}, E\right)$. For $Q\left(F_{K_{1}}, E\right) \supset[f]$, we have that $\mu_{f}$ is extremal in $[f]$.

For $0<k<1$, let $\mu(x, y)=k$ for $(x, y) \in S$ and let

$$
\nu(x, y)= \begin{cases}k, & (x, y) \in S, x \geq 0, \\ 0, & (x, y) \in S, x<0 .\end{cases}
$$

Then both $\mu$ and $\nu$ are extremal.

Let $A=[0], B=[\mu]$, and $C=[\nu]$. We have two geodesic segments,

$$
\gamma_{A B}=\{[t \mu] \mid 0 \leq t \leq 1\} \quad \text { and } \quad \gamma_{A C}=\{[t \nu] \mid 0 \leq t \leq 1\} .
$$

Let

$$
\eta=\mu_{f^{\mu} \circ\left(f^{\nu}\right)^{-1}}=\frac{\mu-\nu}{1-\mu \bar{\nu}} \frac{f_{z}^{\nu}}{\overline{f_{z}^{\nu}}} \circ\left(f^{\nu}\right)^{-1}= \begin{cases}0, & (x, y) \in S, x \geq 0, \\ k, & (x, y) \in S, x<0 ;\end{cases}
$$

then $\eta$ is extremal (in $\left[f^{\mu} \circ\left(f^{\nu}\right)^{-1}\right]$ ). So we have the geodesic

$$
\gamma_{C B}=\left\{\left[f^{t \eta} \circ f^{\nu}\right] \mid 0 \leq t \leq 1\right\} .
$$

Thus the geodesic segments $\gamma_{A B}, \gamma_{A C}$, and $\gamma_{C B}$ form a triangle $\triangle A B C$ in the form of (3). We will show that the sum of the three inner angles of this triangle is $\pi$.

First, we calculate $\angle A$. Note that $\gamma_{A B}(r)=\left[\frac{r}{k} \mu\right]$ and $\gamma_{A C}(r)=\left[\frac{r}{k} \nu\right]$. We have

$$
\mu_{f \frac{r}{k} \mu \circ\left(f \frac{r}{k} \nu\right)^{-1}}=\frac{\frac{r}{k} \mu-\frac{r}{k} \nu}{1-\frac{r}{k} \mu \frac{r}{k} \nu} \frac{f_{z}^{\frac{r}{k} \nu}}{\overline{f_{z}^{\frac{r}{k}} \nu}} \circ\left(f^{\frac{r}{k} \nu}\right)^{-1}= \begin{cases}0, & (x, y) \in S, x \geq 0, \\ r, & (x, y) \in S, x<0 .\end{cases}
$$

Following Lemma 8 $\mu_{f \frac{r}{k} \mu_{\circ}\left(f^{\frac{r}{k}}\right)^{-1}}$ is extremal in $\left[f^{\frac{r}{k}} \mu^{\circ} \circ\left(f^{\frac{r}{k} \nu}\right)^{-1}\right]$. So we have that

$$
d_{T}\left(\gamma_{A C}(r), \gamma_{A B}(r)\right)=\frac{1}{2} \log \frac{1+r}{1-r}
$$

This implies that

$$
\begin{aligned}
\angle A & =2 \arcsin \left\{\frac{1}{2} \lim _{r \rightarrow 0} \frac{d_{T}\left(\gamma_{A B}(r), \gamma_{A C}(r)\right)}{r}\right\} \\
& =2 \arcsin \left\{\frac{1}{2} \lim _{r \rightarrow 0} \frac{\frac{1}{2} \log \frac{1+r}{1-r}}{r}\right\}=\frac{\pi}{3} .
\end{aligned}
$$

Secondly, we calculate $\angle C$. Let $r^{\prime}=\frac{k-r}{1-k r}$ then

$$
\begin{aligned}
d_{T}\left(\gamma_{C A}(r), A\right) & =d_{T}(C, A)-d_{T}\left(\gamma_{C A}(r), C\right) \\
& =\frac{1}{2} \log \frac{1+k}{1-k}-\frac{1}{2} \log \frac{1+r}{1-r} \\
& =\log \frac{1+r^{\prime}}{1-r^{\prime}} \\
& =d_{T}\left(\gamma_{A C}\left(r^{\prime}\right), A\right) .
\end{aligned}
$$

Thus

$$
\gamma_{C A}(r)=\gamma_{A C}\left(r^{\prime}\right)=\left[\frac{r^{\prime}}{k} \nu\right]
$$


By the definition of $\gamma_{C B}$, we have

$$
\gamma_{C B}(r)=\left[f^{\frac{r}{k} \eta} \circ f^{\nu}\right]=\left[\eta^{\prime}\right]
$$

where

$$
\eta^{\prime}=\mu_{f \frac{r}{k} \eta \circ f^{\nu}}=\frac{\frac{r}{k} \eta \circ f^{\nu}(z) \frac{\overline{f_{z}^{\nu}}}{f_{z}^{\nu}}+\nu}{1+\bar{\nu} \frac{r}{k} \eta \circ f^{\nu}(z) \frac{\overline{f_{z}^{\nu}}}{f_{z}^{\nu}}}= \begin{cases}k, & (x, y) \in S, x \geq 0 \\ r, & (x, y) \in S, x<0 .\end{cases}
$$

So we have

$$
\mu_{f^{\frac{r^{\prime}}{k} \nu} \circ\left(f^{\eta^{\prime}}\right)^{-1}}=\frac{\frac{r^{\prime}}{k} \nu-\eta^{\prime}}{1-\frac{r^{\prime}}{k} \nu \overline{\eta^{\prime}}} \frac{f_{z}^{\eta^{\prime}}}{f_{z}^{\eta^{\prime}}} \circ\left(f^{\eta^{\prime}}\right)^{-1}= \begin{cases}\frac{r^{\prime}-k}{1-r^{\prime} k}, & (x, y) \in S, x \geq 0, \\ -r, & (x, y) \in S, x<0 .\end{cases}
$$

Since

$$
\left|\frac{r^{\prime}-k}{1-r^{\prime} k}\right|=\frac{k-\frac{k-r}{1-k r}}{1-\frac{k-r}{1-k r} k}=\frac{k(1-k r)-(k-r)}{(1-k r)-k(k-r)}=r
$$

and since $\mu_{f \frac{r^{\prime}}{k} \circ \circ\left(f^{\eta^{\prime}}\right)^{-1}}$ is extremal in $\left[f^{\frac{r^{\prime}}{k} \nu} \circ\left(f^{\eta^{\prime}}\right)^{-1}\right]$, the Teichmüller distance

$$
\begin{aligned}
d_{T}\left(\gamma_{C B}(r), \gamma_{C A}(r)\right) & =\frac{1}{2} \inf _{\kappa \in\left[f^{\frac{r^{\prime}}{k}} \circ \circ\left(f \eta^{\prime}\right)^{-1}\right]} \log \frac{1+\|\kappa\|_{\infty}}{1-\|\kappa\|_{\infty}} \\
& =\frac{1}{2} \log \frac{\left\|\mu_{f^{\frac{r^{\prime}}{k}}{ }^{\prime} \circ\left(f^{\eta^{\prime}}\right)^{-1}}\right\|_{\infty}}{1-\left\|\mu_{f \frac{r^{\prime}}{k} \nu} \circ\left(f^{\eta^{\prime}}\right)^{-1}\right\|_{\infty}} \\
& =\frac{1}{2} \log \frac{1+r}{1-r} .
\end{aligned}
$$

By the same computation as (5), we have

$$
\angle C=\frac{\pi}{3} .
$$

Finally, we calculate $\angle B$. Similarly, $\gamma_{B A}(r)=\gamma_{A B}\left(r^{\prime}\right)=\left[\frac{r^{\prime}}{k} \mu\right]$. We also have

$$
\gamma_{B C}(r)=\gamma_{C B}\left(r^{\prime}\right)=\left[f^{\frac{r^{\prime}}{k} \eta} \circ f^{\nu}\right]=\left[\eta^{\prime \prime}\right]
$$

where

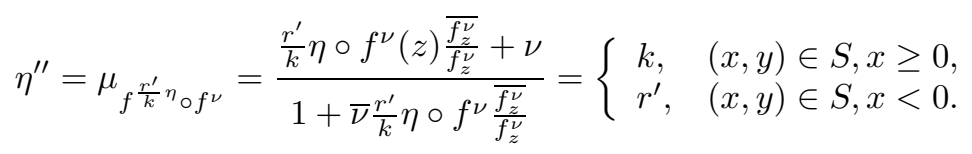

Thus

$$
\tau=\mu_{f^{\frac{r^{\prime}}{k}} \mu_{\circ}\left(f^{\eta^{\prime \prime}}\right)^{-1}}=\frac{\frac{r^{\prime}}{k} \mu-\eta^{\prime \prime}}{1-\frac{r^{\prime}}{k} \mu \overline{\eta^{\prime \prime}}} \frac{\partial_{z} f^{\eta^{\prime \prime}}}{\partial_{z} f^{\eta^{\prime \prime}}} \circ\left(f^{\eta^{\prime \prime}}\right)^{-1}= \begin{cases}\frac{r^{\prime}-k}{1-r^{\prime} k}, & (x, y) \in S, x \geq 0, \\ 0, & (x, y) \in S, x<0 .\end{cases}
$$


So $\tau$ is extremal in $\left[f^{\frac{r^{\prime}}{k}} \mu \circ\left(f^{\eta^{\prime \prime}}\right)^{-1}\right]$ and the Teichmüller distance

$$
\begin{aligned}
d_{T}\left(\gamma_{B C}(r), \gamma_{B A}(r)\right) & =\frac{1}{2} \inf _{\kappa \in\left[f^{\frac{r^{\prime}}{k}} \mu_{\left.\circ\left(f^{\eta^{\prime \prime}}\right)^{-1}\right]} \log \frac{1+\|\kappa\|_{\infty}}{1-\|\kappa\|_{\infty}}\right.} \\
& =\frac{1}{2} \log \frac{\mu_{f^{\frac{r^{\prime}}{k}} \mu_{\circ}\left(f^{\eta^{\prime \prime}}\right)^{-1}} \|_{\infty}}{1-\left\|\mu_{f^{\frac{r^{\prime}}{k}} \mu_{\circ}\left(f^{\eta^{\prime \prime}}\right)-1}\right\|_{\infty}} \\
& =\frac{1}{2} \log \frac{1+\left|\frac{r^{\prime}-k}{1-r^{\prime} k}\right|}{1-\left|\frac{r^{\prime}-k}{1-r^{\prime} k}\right|}=\frac{1}{2} \log \frac{1+r}{1-r} .
\end{aligned}
$$

Using this and the same computation as in (5), we have

$$
\angle C=\frac{\pi}{3} \text {. }
$$

Put (5), (6), and (7) together, we get

$$
\angle A+\angle B+\angle C=\pi .
$$

This completes the proof of Theorem 4.

\section{Proof of Theorem 5}

For $0<k<1$, let $\mu(x, y)=k$ for $(x, y) \in S$. Define

$$
\nu(x, y)= \begin{cases}-k, & (x, y) \in S, x \geq 0 \\ 0, & (x, y) \in S, x<0 .\end{cases}
$$

Then $\mu$ and $\nu$ are extremal.

Let $A=[0], B=[\mu]$ and $C=[\nu]$. Then

$$
\gamma_{A B}=\{[t \mu] \mid 0 \leq t \leq 1\} \quad \text { and } \quad \gamma_{A C}=\{[t \nu] \mid 0 \leq t \leq 1\}
$$

are geodesic segments. Let

$$
\eta=\mu_{f^{\mu} \circ\left(f^{\nu}\right)^{-1}}=\frac{\mu-\nu}{1-\mu \bar{\nu}} \frac{f_{z}^{\nu}}{\overline{f_{z}^{\nu}}} \circ\left(f^{\nu}\right)^{-1}= \begin{cases}\frac{2 k}{1+k^{2}}, & (x, y) \in S, x \geq 0, \\ k, & (x, y) \in S, x<0\end{cases}
$$

then $\eta$ is extremal (in $\left[f^{\mu} \circ\left(f^{\nu}\right)^{-1}\right]$ ). We have the geodesic segment

$$
\gamma_{C B}=\left\{\left[f^{t \eta} \circ f^{\nu}\right] \mid 0 \leq t \leq 1\right\} .
$$

Three geodesic segments $\gamma_{A B}, \gamma_{A C}$, and $\gamma_{C B}$ form a triangle $\triangle A B C$ in the form of (3). We will prove that the sum of inner angles of this triangle is greater than $\pi$.

First, we calculate $\angle A$. It is easy to see that $\gamma_{A B}(r)=\left[\frac{r}{k} \mu\right]$ and $\gamma_{A C}(r)=\left[\frac{r}{k} \nu\right]$. Then

$$
\tau=\mu_{\left.f \frac{r}{k} \mu_{\circ\left(f \frac{r}{k} \nu\right.}\right)^{-1}}=\frac{\frac{r}{k} \mu-\frac{r}{k} \nu}{1-\frac{r}{k} \mu \frac{r}{k} \nu} \frac{f_{z}^{\frac{r}{k} \nu}}{f_{z}^{\frac{r}{k} \nu}} \circ\left(f^{\frac{r}{k} \nu}\right)^{-1}= \begin{cases}\frac{2 r}{1+r^{2}}, & (x, y) \in S, x \geq 0, \\ r, & (x, y) \in S, x<0 .\end{cases}
$$

Since $\frac{2 r}{1+r^{2}}>r$ and since $\tau$ is extremal in $\left[f^{\frac{r}{k} \mu} \circ\left(f^{\frac{r}{k}} \nu\right)^{-1}\right]$, we can calculate the Teichmüller distance as

$$
d_{T}\left(\gamma_{A C}(r), \gamma_{A B}(r)\right)=\frac{1}{2} \log \frac{1+\frac{2 r}{1+r^{2}}}{1-\frac{2 r}{1+r^{2}}}=\log \frac{1+r}{1-r}
$$


Thus we have

$$
\begin{aligned}
\angle A & =2 \arcsin \left\{\frac{1}{2} \lim _{r \rightarrow 0} \frac{d_{T}\left(\gamma_{A B}(r), \gamma_{A C}(r)\right)}{r}\right\} \\
& =2 \arcsin \left\{\frac{1}{2} \lim _{r \rightarrow 0} \frac{\log \frac{1+r}{1-r}}{r}\right\} \\
& =2 \arcsin 1=\pi .
\end{aligned}
$$

Secondly, we calculate $\angle C$. Similarly to the proof of Theorem 4 we know that

$$
\gamma_{C A}(r)=\gamma_{A C}\left(r^{\prime}\right)=\left[\frac{r^{\prime}}{k} \nu\right]
$$

and

where

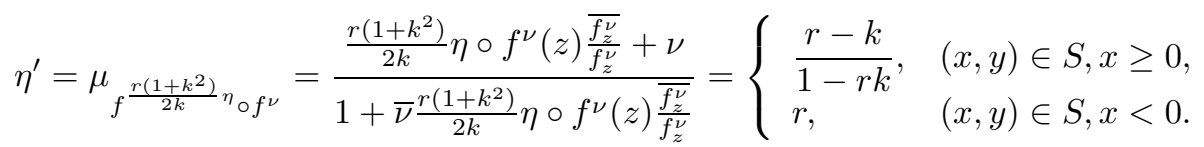

Thus

$$
\eta^{\prime \prime}=\mu_{f^{\frac{r^{\prime}}{k}} \circ \circ\left(f^{\eta^{\prime}}\right)^{-1}}=\frac{\frac{r^{\prime}}{k} \nu-\eta^{\prime}}{1-\frac{r^{\prime}}{k} \nu \overline{\eta^{\prime}}} \frac{f_{z}^{\eta^{\prime}}}{f_{z}^{\eta^{\prime}}} \circ\left(f^{\eta^{\prime}}\right)^{-1}= \begin{cases}0, & (x, y) \in S, x \geq 0, \\ -r, & (x, y) \in S, x<0 .\end{cases}
$$

So $\eta^{\prime \prime}$ is extremal in $\left[f^{\frac{r^{\prime}}{k}} \nu \circ\left(f^{\eta^{\prime}}\right)^{-1}\right]$ and the Teichmüller distance

$$
d_{T}\left(\gamma_{C B}(r), \gamma_{C A}(r)\right)=\frac{1}{2} \log \frac{1+r}{1-r} .
$$

Using this and the same computation as in (5), we have

$$
\angle C=\frac{\pi}{3} \text {. }
$$

Then (8) and (9) already imply that

$$
\angle A+\angle B+\angle C>\pi \text {. }
$$

This completes the proof.

\section{REFERENCES}

[1] V. Božin, N. Lakic, V. Marković, and M. Mateljević, Unique extremality, J. Anal. Math. 75 (1998), 299-338, DOI 10.1007/BF02788704. MR1655836 (2000a:30045)

[2] Clifford J. Earle and Zhong Li, Isometrically embedded polydisks in infinite dimensional Teichmüller spaces, J. Geom. Anal. 9 (1999), no. 1, 51-71, DOI 10.1007/BF02923088. MR.1760720 (2001f:32022)

[3] C. J. Earle, I. Kra, and S. L. Krushkal', Holomorphic motions and Teichmüller spaces, Trans. Amer. Math. Soc. 343 (1994), no. 2, 927-948, DOI 10.2307/2154750. MR1214783(94h:32035)

[4] A. Fletcher and V. Markovic, Quasiconformal maps and Teichmüller theory, Oxford Graduate Texts in Mathematics, vol. 11, Oxford University Press, Oxford, 2007. MR2269887 (2007g:30001)

[5] F. P. Gardiner and N. Lakic, Quasiconformal Teichmüller theory, Math Surveys and Monographs, vol. 76, American Mathematical Society, Providence, 2000. MR.1730906

[6] Richard S. Hamilton, Extremal quasiconformal mappings with prescribed boundary values, Trans. Amer. Math. Soc. 138 (1969), 399-406. MR0245787 (39 \#7093)

[7] Nikola Lakic, Strebel points, Lipa's legacy (New York, 1995), Contemp. Math., vol. 211, Amer. Math. Soc., Providence, RI, 1997, pp. 417-431, DOI 10.1090/conm/211/02832. MR1476999 (99c:32027) 
[8] Olli Lehto, Univalent functions and Teichmüller spaces, Graduate Texts in Mathematics, vol. 109, Springer-Verlag, New York, 1987. MR867407 (88f:30073)

[9] Li Zhong, Nonuniqueness of geodesics in infinite-dimensional Teichmüller spaces, Complex Variables Theory Appl. 16 (1991), no. 4, 261-272. MR.1105200 (92c:32024)

[10] Zhong Li, Nonuniqueness of geodesics in infinite-dimensional Teichmüller spaces. II, Ann. Acad. Sci. Fenn. Ser. A I Math. 18 (1993), no. 2, 355-367. MR1234739 (94h:32037)

[11] Zhong Li, Non-convexity of spheres in infinite-dimensional Teichmüller spaces, Sci. China Ser. A 37 (1994), no. 8, 924-932. MR1323829 (96d:32022)

[12] Li Zhong, A note on geodesics in infinite-dimensional Teichmüller spaces, Ann. Acad. Sci. Fenn. Ser. A I Math. 20 (1995), no. 2, 301-313. MR1346814 (96h:30087)

[13] Li Zhong, Closed geodesics and non-differentiability of the metric in infinite-dimensional Teichmüller spaces, Proc. Amer. Math. Soc. 124 (1996), no. 5, 1459-1465, DOI 10.1090/S00029939-96-03164-4. MR1301053 (96g:32036)

[14] Zhong $\mathrm{Li}$ and $\mathrm{Yi} \mathrm{Qi}$, Fundamental inequalities of Reich-Strebel and triangles in a Teichmüller space, Quasiconformal mappings, Riemann surfaces, and Teichmüller spaces, Contemp. Math., vol. 575, Amer. Math. Soc., Providence, RI, 2012, pp. 283-297, DOI 10.1090/conm/575/11398. MR2933905

[15] S. L. Krushkal, Extremal quasiconformal mappings, Siberian Math. J. 10 (1969), 411-418.

[16] Edgar Reich, Extremal quasiconformal mappings of the disk, Handbook of complex analysis: geometric function theory, Vol. 1, North-Holland, Amsterdam, 2002, pp. 75-136, DOI 10.1016/S1874-5709(02)80005-1. MR.1966190(2004c:30036)

[17] Edgar Reich and Kurt Strebel, Extremal quasiconformal mappings with given boundary values, Contributions to analysis (a collection of papers dedicated to Lipman Bers), Academic Press, New York, 1974, pp. 375-391. MR0361065 (50 \#13511)

[18] Kurt Strebel, On the extremality and unique extremality of quasiconformal mappings of a parallel strip, Rev. Roumaine Math. Pures Appl. 32 (1987), no. 10, 923-928. MR.924137 (89f:30042)

[19] G. Yao, A binary infinitesimal form of Teichmüller metric, to appear.

Department of Applied Mathematics, Nanjing University of Science and Technology, Nanjing, 210094, People's Republic of China

E-mail address: jinhuafan@hotmail.com

mathematics9 Department, Queens College of CUNY, 65-30 Kissena Blvd, Flushing, New York 11367 - and - Ph.D. Program in Mathematics, Graduate Center of CUNY, 365 Fifth Avenue, New York, New York 10016

E-mail address: yunping.jiang@qc.cuny.edu 